\title{
Information-theoretic View of the Variation of the Gravitational Constant
}

\author{
Subhash Kak \\ Oklahoma State University, Stillwater
}

\begin{abstract}
Many recent theories speak of the spatial and temporal variations of the fundamental "constants", and experiments have been performed to determine the variation. It has also been proposed that the variation of these constants is related to the strength of the gravitational potential whose changes, therefore, assume special significance. Here we examine potential change in $\mathrm{G}$ from the information-theoretic perspective of noninteger dimensionality and determine a relationship with respect to time. We show that the rate of change using this method is of the same order as estimated using other approaches.
\end{abstract}

\section{Introduction}

The power of information theory as an analytical tool derives from the fact that it can be potentially applied to systems of any size [1] and that it allows us to consider evolution of the system in relation to a suitably chosen informational measure. It should be possible, therefore, to apply information theory to the universe, the largest system of all, and investigate the problem of whether the fundamental constants of nature change with time, a problem that is of interest from the perspectives of science, engineering, and natural systems [2][3].

The Large Numbers Hypothesis (LNH) of Dirac [4][5] speaks of the possibility of variation of the fundamental constants with respect to the age of the universe. The logic behind this idea is that constants cannot be independent of the structure of the universe and as the universe has evolved, so must the constants, for if the laws -- and constants -- were independent of the universe, then they would constitute a separate non-physical reality needing to be explained. Dirac argued that the age of the Universe may be related to atomic constants that yield the large dimensionless number $t \sim 10^{39}$ or its powers. Other proposals, reviewed in [3], suggest that the fundamental constants should vary with the strength of the gravitational field.

Specifically, in LNH, the electric force between the electron and the proton in the hydrogen atom is $\frac{e^{2}}{r^{2}}$, and the gravitational force between them is $\frac{G m_{p} m_{e}}{r^{2}}$. Their ratio is the dimensionless number $\frac{e^{2}}{G m_{p} m_{e}} \sim 10^{39}$ that should increase in 
proportion to the age of the universe. Since $\mathrm{G}$ is in the denominator, this means that $\mathrm{G}$ must decrease, and be proportional to $t^{-1}$.

The value of the gravitational constant is $\mathrm{G}=6.67430(15) \times 10^{-11} \mathrm{~m}^{3} \cdot \mathrm{kg}^{-1}$. $\mathrm{s}^{-2}$ and its relative standard uncertainty fixed by the 2018 CODATA is 2.2 $\times 10^{-5}$. The variation of the gravitational constant does not affect the local physics for gravity is much smaller force compared to the other forces and it is difficult to gauge its variation in geological time.

The precision in the measurement of $G$ is relatively low compared to the measurement of other constants [6], and there is at least one report of a periodic variation of its value [7], with a period of $(5.899+/-0.062)$ per year with a small amplitude of $(1.619+/-0.103) \times 10^{-14} \mathrm{~m}^{3} \mathrm{~kg}^{-1} \mathrm{~s}^{-2}$. Various types of bounds on its rate of variation have been proposed. For example, estimated constraints on the variation with Type 1a Supernovae yield a variation of $10^{-10} \mathrm{yr}^{-1}$ over the last nine billion years [8], whereas $\mathrm{BBN}$ constraints are estimated to yield $\dot{G} /_{G} \sim 10^{-12} \mathrm{yr}^{-1}[9]$, and using observations of merging binary neutron stars one gets the estimate to be $-7 \times 10^{-9} \mathrm{yr}^{-1} \leq \dot{G} /{ }_{G} \leq 5 \times 10^{-8} \mathrm{yr}^{-1}[10]$. There exists a huge amount of literature on the varying constants problem and for a very recent review, see the 2021 paper [11].

The information-theoretic perspective of noninteger dimensionality and its relationship to gravitational force has been considered recently by the author [12][13][14]. If the increase of the intrinsic dimensionality of the system is seen as the driver of the evolution, one can then determine the functional form for $\mathrm{G}$ (as in [15]). In the present paper, we take the next step of mapping the variation of $\mathrm{G}$ to one with respect to time by assuming that dimensionality and time have a uniform relationship. Three different models of the relationship between $G$ and time are considered. The estimates obtained are compared with those from recent experiments and shown to be of the same order of magnitude or smaller.

\section{Linked variability of fundamental constants}

The possible variation of $\mathrm{G}$ is sometimes seen together with that of the variation of the dimensionless fine-structure constant, $\alpha$, which relates the strength of the electromagnetic interaction between an elementary charged particle of charge e with the electromagnetic field according to the formula $4 \pi \varepsilon_{0} \hbar c \alpha=\mathrm{e}^{2}$, where $\varepsilon_{0}$ is permittivity in vacuum, $\hbar$ is the reduced Planck's constant, and c is the speed of light in vacuum. Jordan [16] proposed a theory in which the constants become dynamical fields and both $\mathrm{G}$ and $\alpha$ can vary. It has been proposed that whereas for zero-energy scale of the electron mass $\alpha \sim 1 / 137.036$, at higher 
energies it is effectively about $1 / 127$ [17]. It has further been estimated to have increased slightly over the past few billion years [18].

It is the usual unstated assumption that laws of physics do not differ from one point to another in spacetime. But they can be operationally different if Mach's principle is applied as was done by Brans and Dicke [19][20], by replacing the gravitational constant by a scalar field that varies both spatially and in time.

A meaningful test on the time-variation of $\mathrm{G}$ would require comparison with some non-gravitational force to obtain a dimensionless quantity, as in the ratio of the gravitational force to the electrostatic force between two electrons, which, in turn, is related to $\alpha$ [21].

The 2018 CODATA value [22] of $\alpha$ is 7.2973525693(11) $\times 10^{-3}$. Webb et $a l$. [23] found that the spectra were consistent with a slight increase in it over the last $10-12$ billion years. Specifically, it was found that $\frac{\Delta \alpha}{\alpha}=(-5.7 \pm$ 1.0) $\times 10^{-6}$.

Observations of the redshift $\mathrm{z}=7.085$ quasar $\mathrm{J} 1120+0641$ have been used to search for variations of $\alpha$, over the redshift range 5.5 to 7.1. When new measurements were combined with a large existing sample of lower redshift measurements, it was found that a possible spatial variation of $\Delta \alpha / \alpha$ is marginally superior over a no-variation model at the $3.7 \sigma$ level. In observations of the quasar of the universe at only 0.8 billion years old a spatial variation preferred over a no-variation model at the 3.7 $\sigma$ level [24]. Other limits in the range of $10^{-7}$ are related to the measurements of atomic frequencies on the distance from Sun [2]. Summarizing, the variation is seen in the range of $\sim 10^{-7}$ to $10^{-6}$.

\section{Dynamics arising from noninteger dimensionality}

Now we come to the information theoretic view of dimensions of space. One may see it as representation of data in the experienced world from the perspective of fractal structures [25], optimal representation of data [26][27], and logic [1]. Equivalently, one may adopt the complementary view that attraction is a consequence of the fact that space is not fully "packed", as in a fractal structure [12][13]. In other words, if the space were to be integer valued, such a force of attraction will not exist.

If one were to view this attraction as gravitational force, it will begin to rise from the dimensionality, $d$, equal to 2 and peak at some value of $\mathrm{d}$ between 2 
and 3 (as in [13]). Assuming symmetry, we can take the value of peak to be given by $d=2.5$ and represent this maximum by $G_{m}$.

The idea of such information-based dynamics should apply to evolution at all scales and is of potential relevance in the design and investigation of the properties of metamaterials [28][29][30].

Now consider the universe as the evolving system. It is proposed that the evolution of $\mathrm{G}$ has occurred in a uniform manner with respect to the cosmological time that varies uniformly with $d$.

We will look at three different functions: quadratic $G_{1}$, sinusoidal $G_{2}$, and triangular linear $G_{3}$.

The simplest quadratic relationship between dimensionality $d$ and $G$, is given by [13][15]:

$$
G_{1}(d)=4 G_{m}\left(5 d-d^{2}-6\right)
$$

The sinusoidal gravitational potential distribution is:

$$
G_{2}(d)=G_{m} \sin (\pi d-2 \pi)
$$

Finally, the triangular linear function is:

$$
G_{3}(d)=\left\{\begin{array}{c}
2 G_{m}(d-2), d \leq 2.5 \\
2 G_{m}(-d+3), d>2.5
\end{array}\right.
$$

Each of these functions has peak of $G_{m}$ at $d=2.5$.

We know from astronomical records that the expansion of the universe began to accelerate 9.8 billion years after the big bang (time 0 for our equation) [31][32], and we propose that this happened as the drag of the gravitational attraction opposing the expansion due to the potential singularity of time $=0$ began to weaken. Assuming the relationship to be a simple one, this indicates that we can take the peak $\mathrm{G}=G_{m}$ to have occurred at $t=9.8$ billion years.

By change of units $t=a d+b$, we get an equation for change in $\mathrm{G}$ with respect to time in billions of years. To solve for $a$ and $b$, we use the conditions $d=2.5$ corresponds to $t=9.8$, and, of course, that $d=2$ corresponds to $t=0$. 
Solving, we get that $a=19.6$ and $b=-39.2$. The optimal value of $d=e$ is obtained for $t=a e+b=14.078$ billion years.

Considering that the estimated age of the universe is 13.77 billion years, the above expressions imply that the gravitational constant will plateau in about another 300 million years.

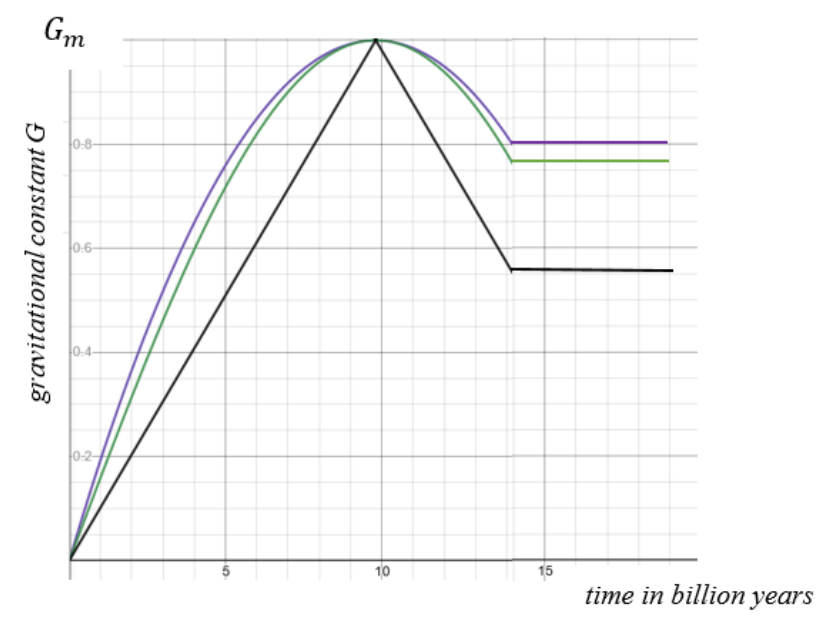

Figure 1. Three different models relating $\mathrm{G}$ to time till reaching optimal value (purple, quadratic; green, sinusoidal; black, zig-zag linear)

In Figure 1, it is assumed that once the optimal point is reached there is no further evolution of dimensionality and consequently $\mathrm{G}$ will become fixed. This has implications for the understanding of the end of the universe. Current scenarios (e.g [33][34]) assume that the acceleration of the expansion will continue and the end will be a cold death of the universe. According to Figure 1 , after the gravitational constant $\mathrm{G}$ attains the value corresponding to optimal dimensionality of $e$, it could at some point become stronger than the force related to the potential that led to the explosion at cosmic time $t=0$. This leaves open the possibility that the acceleration will slow down and then reverse, thus forestalling the cold freeze end of the universe.

Now consider the three cases individually.

Case 1. $\boldsymbol{G}_{\mathbf{1}}(\boldsymbol{t})$

$$
G_{1}(t)=\frac{G_{m}}{96.04}\left(19.6 t-t^{2}\right)
$$

The rate of change of $d$ is easily obtained by differentiating the time expression associated with G. We have: 


$$
\frac{d G}{d t}=\frac{G_{m}}{96.04}(19.6-2 t)
$$

At the current time of $t=13.77$ billion years it is changing at a rate of

$$
\frac{\dot{G}}{G} \approx-0.1 \times 10^{-9} \text { per year }
$$

where the $10^{-9}$ in this expression and others that follow comes from the fact that the original expression is in the units of billion years. This is smaller than the uncertainty of the 2018 CODATA.

The strength of $\mathrm{G}$ at the optimal value of $d=e$ is:

$$
\left.G_{1}(14.078)\right)=\frac{4 G_{m}\left(19.6 \times 14.07-14.07^{2}\right)}{384.16}=0.81 G_{m}
$$

At the optimal point, $\mathrm{G}$ will have a value that is $81 \%$ of its maximum.

It is only sometime after that the expansion will slow down and once the potential driving the expansion weakens that the accelerating expansion will slow down and subsequently reverse.

Case 2. $\boldsymbol{G}_{\mathbf{2}}(\boldsymbol{t})$

In $G_{2}(d)=G_{m} \sin (\pi d-2 \pi)$, let $t=a d+b$ and using the values of a and $\mathrm{b}$ that have already been discussed

$$
G_{2}(t)=G_{m} \sin \left(\frac{(t+39.2) \pi}{19.6}-2 \pi\right)
$$

Or,

$$
G_{2}(t)=G_{m} \sin (0.051 \pi t)
$$

The optimal value of $d=e$ is reached when $t=a e+b$ or for $t=14.07$ billion years. the value of $\mathrm{G}$ will be

$$
G_{2}(t=14.078)=G_{m} \sin (0.051 \pi t)=0.775 G_{m}
$$

The sine function gives a value at $d=e$, that is $77.5 \%$ of the peak value. 


$$
\frac{\dot{G}}{G} \approx-0.13 \times 10^{-9} \text { per year }
$$

Case 3. $\boldsymbol{G}_{\mathbf{3}}(\boldsymbol{t})$

Similar to the other two cases, using formula (3), we get

$$
G_{3}(t)=\left\{\begin{array}{c}
0.102 G_{m} t, t \leq 9.8 \\
2 G_{m}(1-0.051 t), d>9.8
\end{array}\right.
$$

The optimal value of $d=e$, for $t=14.078$ billion years, turns out to be:

$$
G_{3}(t=14.078)=2 G_{m}(1-0.051 \times 14.078)=0.564 G_{m}
$$

The value at optimal $d$ for this case is substantially lower (56.4\%) than for the cases of the quadratic (81\%) and the sinusoidal functions $(77.5 \%)$, respectively.

The rate of change for this case is:

$$
\frac{\dot{G}}{G} \approx-0.180 \times 10^{-9} \text { per year }
$$

The rate of change for each of these is comparable to the estimates using current experimental schemes [8][9][10] that were reviewed in Section 2.

\section{Conclusions}

If evolution is seen as driven by the increase of the intrinsic dimensionality of the system, one can then quantify the value of changing $G$. This paper mapped the variation of $\mathrm{G}$ to one with respect to time by assuming that dimensionality and time have a uniform relationship. Three different models of the relationship between $\mathrm{G}$ and time were considered. The estimates were compared with those from recent experiments and it was found that they are well within the uncertainty estimates for both for gravitational and fine-structure constants. Furthermore, the evolution indicated by our models can forestall the scenario of the eventual cold death of the universe.

The result of this paper supports the view, first proposed in [12], that space is associated with noninteger dimensionality and strengthens the plausibility of the dimensionality view of the change in the gravitational constant. 


\section{REFERENCES}

1. Kak, S. (2021) Logic of representation and information. https://www.techrxiv.org/articles/preprint/Logic_of_Representation_and_I nformation/13601939

2. Flambaum, V.V. (2007). Variation of fundamental constants: theory and observations. International Journal of Modern Physics A 22, 4937-4950

3. Uzan, J.-P. (2011). Varying constants, gravitation and cosmology. Living Reviews in Relativity 14, 2.

4. Dirac, P. A. M. (1938). A New Basis for Cosmology. Proceedings of the Royal Society of London A. 165, 199-208.

5. Dirac, P. A. M. (1974). Cosmological models and the Large Numbers hypothesis. Proc. Royal Society London A 338, 439-446.

6. Reasenberg, R.D. (1983). The constancy of $G$ and other gravitational experiments. Philosophical Transactions of The Royal Society A: Mathematical, Physical and Engineering Sciences, 310, 227-238, 1983.

7. Anderson, J.D. et al. (2015). Measurements of Newton's gravitational constant and the length of day. EPL 110, 10002; arXiv:1504.06604

8. Mould, J., Uddin, S.A. (2014). Constraining a possible variation of $G$ with Type Ia Supernovae. Publications of the Astronomical Society of Australia 31, e015; arXiv:1402.1534

9. Alvey, J. et al. (2020). Improved BBN constraints on the variation of the gravitational constant. Eur. Phys. J. C 80, 148.

10. Vijaykumar, A., and Kapadia, S.J., Ajith, P. (2021). Constraints on the time variation of the gravitational constant using gravitational wave observations of binary neutron stars. Phys. Rev. Lett.126, 141104.

11. Nassif, C. and Amaro de Faria Jr, A.C. (2021). Variation of the fundamental constants over the cosmological time: veracity of Dirac's intriguing hypothesis. arXiv:2102.05039

12. Kak, S. (2020). Information theory and dimensionality of space. Scientific Reports 10, 20733; https://www.nature.com/articles/s41598-020-77855-9

13. Kak, S. (2021). Asymptotic freedom and noninteger dimensionality. Scientific Reports 11, 1-5; https://www.nature.com/articles/s41598-02183002-9

14. Kak, S. (2021). Fractals with optimal information dimension. Circuits Syst. Signal Process. 40.

15. Kak, S. (2021). Information theory of evolutionary stages in noninteger dimensional spaces.

https://www.techrxiv.org/articles/preprint/Information_Theory_of_Evoluti onary_Stages_in_Noninteger_Dimensional_Spaces/13775005 
16. Jordan, P. (1937). Die physikalischen Weltkonstanten. Die Naturwissenschaften 25, 513-517.

17. Fritzsch, H. (2002). Fundamental constants at high energy. Fortschritte der Physik. 50, 518-524

18. Webb, J.K. et al. (1999). Further evidence for cosmological evolution of the fine structure constant. Phys. Rev. Lett 82 884; astro-ph/0012539.

19. Brans, C., and R.H. Dicke (1961). Mach's principle and a relativistic theory of gravitation. Phys. Rev. 124, 925.

20. Dicke, R.H. (1962) Mach's principle and invariance under transformation of units. Phys. Rev. 125, 2163

21. Uzan, J. -P. (2003). The fundamental constants and their variation: Observational status and theoretical motivations, Rev. Mod. Phys. 75, 403

22. Mohr, P. J.; Taylor, B. N.; Newell, D. B. (2019). Fine structure constant. CODATA Internationally recommended 2018 values of the fundamental physical constants. NIST.

23. Murphy, M.T., Webb, J.K., Flambaum, V.V (2003). Further evidence for a variable fine-structure constant from Keck/HIRES QSO absorption spectra. Mon. Not. Roy. Astron. Soc. 345: 609, 2003

24. Wilczynska, M.R. et al. (2020). Four direct measurements of the finestructure constant 13 billion years ago. Science Advances. 6 (17)

25. Kak, S. (2020). Information, representation, and structure. International Conference on Recent Trends in Mathematics and Its Applications to Graphs, Networks and Petri Nets, New Delhi.

26. Kak, S. (2021). The base-e representation of numbers and the power law. Circuits Syst. Signal Process. 40, 490-500; https://link.springer.com/article/10.1007/s00034-020-01480-0

27. Kak, S. (2021). The intrinsic dimensionality of data. Circuits Syst. Signal Process. 40, 2599-2607; https://link.springer.com/article/10.1007/s00034020-01583-8

28. Grima, J. N., Attard, D. \& Gatt, R. (2008). Truss-type systems exhibiting negative compressibility. Phys. Status Solidi B 245, 2405-2414.

29. Lakes, R. S., Lee, T., Bersie, A. \& Wang, Y. C. (2001). Extreme damping in composite materials with negative-stiffness inclusions. Nature 410, 565567.

30. Scheibner, C., Souslov, A., Banerjee, D. et al. (2020). Odd elasticity. Nat. Phys. 16, 475-480.

31. Frieman, J.A., Turner, Michael S., Huterer, D. (2008). Dark energy and the accelerating universe. Annual Review of Astronomy and Astrophysics 46, 385-432.

32. Rubin, D. and Heitlauf, J. (2020). Is the expansion of the universe accelerating? The Astrophysical Journal 894, 68. 
33. Yurov, A.V., Astashenok, A.V. \& González-Díaz, P.F. (2008). Astronomical bounds on a future Big Freeze singularity. Gravit. Cosmol. 14, 205-212.

34. Wetterich, C. (2014). Hot big bang or slow freeze? Physics Letters B, 736, 506-514. 